\title{
FLOW RIPPLE IN EXTERNAL HELICAL GEAR PUMP
}

\author{
P. Osiński", P. Bury ${ }^{* *}$
}

\begin{abstract}
This paper presents a flow ripple model for external helical gear pumps. The model is for pumps with and without intermesh side play. Moreover, selected aspects of the evaluation of the acoustic properties of prototype low-noise gear pumps developed in collaboration with the firm PHS HYDROTOR S.A. are described. The acoustic characteristics of the prototype pumps are compared with those of commercially available low-noise pumps.
\end{abstract}

Key words: external gear pump, low-noise gear pump, flow ripple, noise reduction, helical gear pump

\section{Introduction}

Displacement pumps, functioning as fluid flow energy generators, are a basic component of hydrostatic drive systems. There are many pump designs, but external gear pumps belong to the most popular ones (Szablowski K., 1997; Osiecki, 2004; Stryczek, 2014).

Most of the current research focuses on system energy efficiency and environmental noise and vibration reduction (Kollek W., 1996; Osinski, 2013; Osiński, 2014). While the efficiency of pumps is quite high for hydraulic equipment, more attention is devoted to the effect of equipment operation on the environment. The main source of noise in pumps, besides the trapped volume and the intermesh, is the flow ripple which generates pressure fluctuations in the whole system. The measure of the flow ripple is pump delivery nonuniformity coefficient $\delta$, which is calculated from the relation:

$$
\delta=\frac{Q_{\max }-Q_{\min }}{Q_{t}}
$$

where: $Q_{\max }$ - is the maximum delivery, $Q_{\min }$ - the minimum delivery, $Q_{t}-$ the medium delivery.

\section{Flow ripple model}

The gear pump belongs to the group of displacement pumps whose principle of operation consists in displacing specified volumes of the fluid from the displacement chambers. In the case of gear pumps, the displacement chambers are the intertooth spaces while the teeth of the counterpart gear are the displacing elements. In order to determine the change in volume $d V$ of the displacement chamber due to tooth entering the intertooth space of the counterpart gear one should carry out a detailed analysis of the geometrical properties of the involute. Analysing this problem on the front face of the intermesh one can determine the change in surface area $(d S)$ caused by the turn of the gears by angle $d \beta$.

As shown in Fig. 1, the initial position at instant $t$ was assumed to be the moment when the teeth's profile is in contact with point $e$ situated at distance $-\overline{C e}$ from intermesh point $C$; positive values were conventionally assumed to be situated to the right of this point. The position of the driving wheel is defined by angle $\beta_{1}$ while the position of the driven wheel is defined by angle $\beta_{2}$.

Piotr Osiński, D.Sc. Eng.: Faculty of Mechanical Engineering, Wroclaw University of Science and Technology, Lukasiewicza 7/9; 50-371 Wroclaw; PL, piotr.osinski@pwr.edu.pl

** Paweł Bury, M.Sc., Eng.: Faculty of Mechanical Engineering, Wroclaw University of Science and Technology, Lukasiewicza 7/9; 50-371 Wroclaw; PL, pawel.bury@pwr.edu.pl 


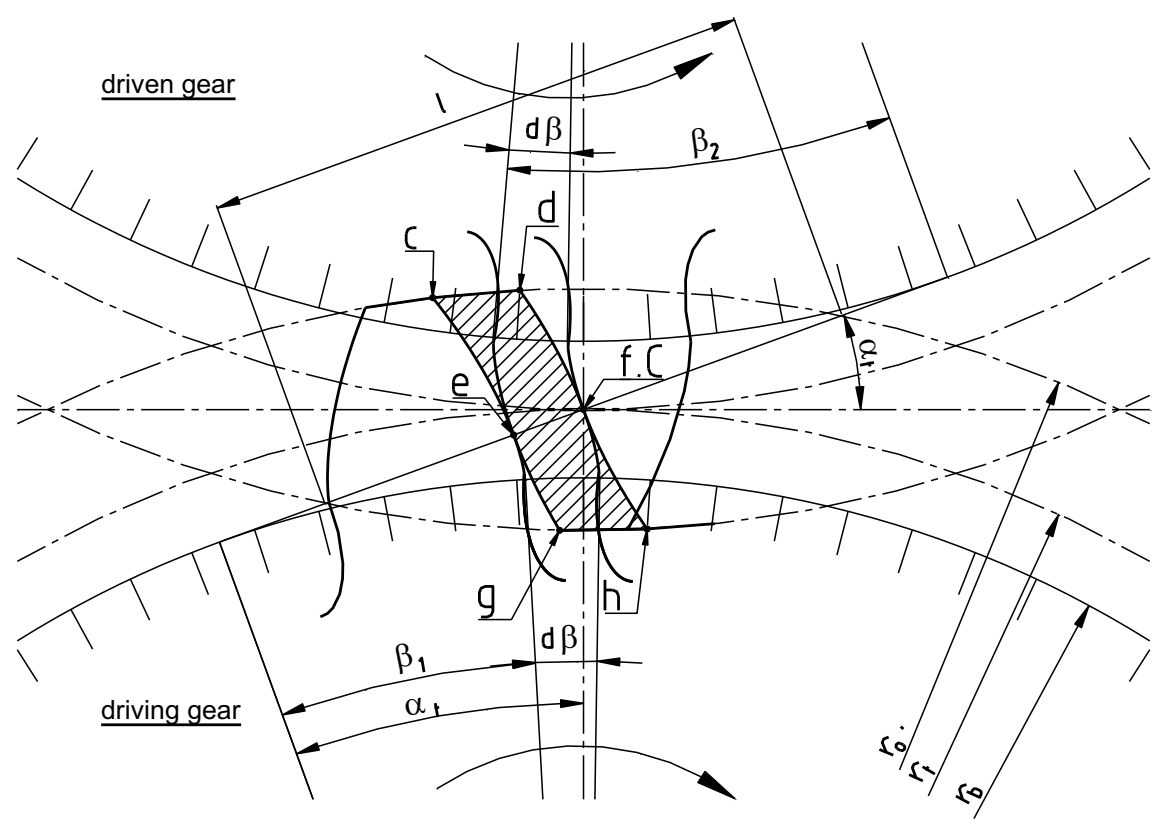

Fig. 1: Geometrical properties of intermesh, where: $c, d, e, f, g, h$-points on the tooth shape, $C$ - pitch point, $\beta_{1}, \beta_{2}$-angular position of gears, $d \beta$-increment of angle in time $d t, \alpha_{t}-$ transverse pressure angle, $r_{o}^{\prime}$-effective outside radius, $r_{t}$ - operating pitch radius, $r_{b}$ - base radius, $l$ length of action.

It was assumed that the rotation of the gears by angle $d \beta$ during instant $d t$ would make the intermesh point shift from point $e$ to point $f$. In the case of helical gears, angular position $\beta_{1}$ of considered cross section $d S$ depends on the position of this cross section relative to the rim's front plane, which can be expressed as $\beta_{1}+\gamma(b)$, where $\gamma$ is the angle of the shift of the cross section situated at distance $b$ relative to the initial cross section. Angle relation $\gamma(b)$ stems from the properties of helical gears and amounts to $\gamma(b)=\frac{\operatorname{tg} \beta_{s}}{r} b$, where $\beta_{s}$ is the angle of inclination of the tooth helix, and $r$ is the pitch radius. The volume of the fluid displaced during time $d t$ will be equal to the integral of the surface area between curves ceg and $d f h$ along wheel width $d b$.

$$
d V_{\beta}=\int_{0}^{b}\left(d S_{c d e f}\left(\beta_{1}+\frac{\operatorname{tg} \beta_{s}}{\mathrm{r}} b\right)+d S_{\text {efgh }}\left(\beta_{1}+\frac{\operatorname{tg} \beta_{s}}{\mathrm{r}} b\right)\right) \mathrm{d} b
$$

Considering the geometrical properties of the involute and the geometry of the gears one gets:

$$
q_{s}=\frac{d V_{\beta}}{d t}=\omega b\left[r^{\prime}{ }_{o}^{2}-r_{t}^{2}-\frac{1}{12}\left(\frac{r_{b} \operatorname{tg} \beta_{s}}{\mathrm{r}} b\right)^{2}\left(s+\frac{r_{b} \operatorname{tg} \beta_{s}}{2 \mathrm{r}} b\right)^{2}\right]
$$

The instantaneous delivery can be related to angle of rotation $\beta$, whereby ultimately one gets:

$$
q_{s}=\frac{d V_{\beta}}{d t}=\omega b\left[r^{\prime}{ }_{o}^{2}-r_{t}^{2}-\frac{1}{12}\left(\frac{r_{b} \operatorname{tg} \beta_{s}}{\mathrm{r}} b\right)^{2}\left(r_{b} \beta-\frac{l}{2}+\frac{r_{b} \operatorname{tg} \beta_{s}}{2 \mathrm{r}} b\right)^{2}\right]
$$

and for the ideal profile:

$$
q_{s}=\frac{d V_{\beta}}{d t}=\omega b\left[r^{\prime}{ }_{o}{ }^{2}-r_{t}^{2}-\frac{1}{12}\left(\frac{r_{b} \operatorname{tg} \beta_{s}}{\mathrm{r}} b\right)^{2}\left(r_{b} \beta-\frac{t_{b}}{4}-\frac{b r_{b} \operatorname{tg} \beta_{s}}{2 \mathrm{r}}+\frac{r_{b} \operatorname{tg} \beta_{s}}{2 \mathrm{r}} b\right)^{2}\right]
$$

Using the above model of instantaneous delivery was calculated from relation reduced from $26.5 \%$ to $19.7 \%$ for a pump with side play and from $5.9 \%$ to $4.2 \%$ for a pump without side play (Bury P., Osiński P., 2015). 


\section{Comparative acoustic tests}

Because of difficulties in direct measuring the flow ripple of displacement pumps it was decided to carry out comparative acoustic measurements. Besides prototype units, low-noise pumps and conventional pumps manufactured by leading firms were subjected to the tests. The tested pumps are briefly described in Table 1.

Tab. 1: List of compared pumps and description of their design.

\begin{tabular}{lll}
\hline No. & Type & Description \\
\hline 1 & Prototype 2PW-SES & Involute helical gear, no backlash \\
\hline 3 & Prototype 2PWR-SE & Involute helical gear, with backlash \\
\hline 4 & Commercial conventional & Involute gear, with backlash \\
\hline 5 & Commercial low noise 1 & Non-involute helical gear, no backlash \\
\hline 6 & Commercial low noise 2 & Involute helical gear, with backlash \\
\hline 7 & Commercial low noise 3 & Non-involute helical gear, no backlash \\
\hline
\end{tabular}

All the hydraulic and acoustic measurements were carried out in the Laboratory of Hydraulic Drives and Machine Vibroacoustics at Wroclaw University of Science and Technology. Measurements of the level of the noise emitted by the gear pumps were carried out in an acoustic diffusion chamber where measurement microphones were set up in eight points. The resultant acoustic pressure level $L_{m}[\mathrm{~dB}]$, sound level $L_{A}[\mathrm{~dB}(\mathrm{~A})]$ and octave spectrum values were read off the microphones and averaged by means of a Brüel\&Kjaer apparatus.

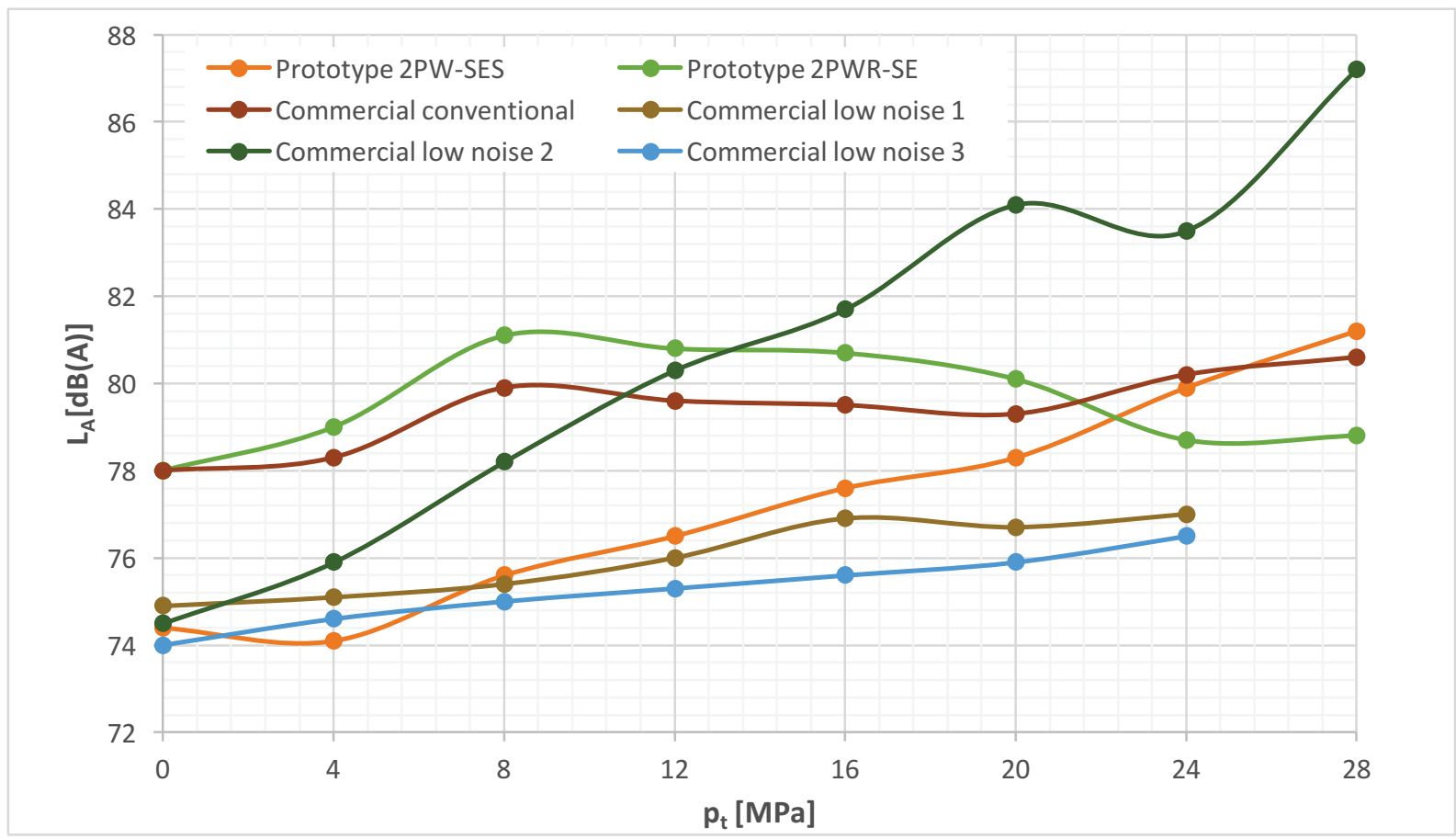

Fig. 2: Sound level $L_{A}$ versus pressure at $1500 \mathrm{rpm}$.

The sound level adjusted to frequency characteristic A $\left(L_{A}-\right.$ Fig. 2$)$ shows that the newly designed pumps have a clear advantage over the conventional pump. Commercial low-noise pumps 1 and 3, with innovative non-involute gears, where the contact between the gears is continuous and no trapped volume forms, emit sound whose level A is considerably lower than that the sound level of the involute gear pumps with backlash (the conventional pump, prototype pump 2PWR-SE and low-noise commercial pump 2). 
Thanks to the use of skew teeth and zero side play in the 2PW-SES series pumps, combined with the innovative profile optimized by means of logical trees, their sound level A was reduced to the level of the sound emitted by the low-noise non-involute gear pumps under low and medium working pressures $p_{t}$. Above $20 \mathrm{MPa}$ the advantage of the non-involute gear pumps is clearer.

\section{Conclusion}

Due to the limited possibilities of measuring rapidly varied flows, it is difficult to experimentally verify the presented flow ripple model. However, the acoustic tests show a clear reduction in the acoustic pressure level directly connected with sound producing vibrations. The use of helical gears results in maximally a few per cent reduction (depending on the pump design) of the delivery nonuniformity coefficient, but considering the other assets of helical gears, such as their lower intermesh noisiness and gentler change in intermesh stiffness, its effects are satisfactory at no significant increase in production costs, as opposed to the costs of manufacture of non-involute gears.

Moreover, the acoustic parameters, especially sound level $L_{A}$, are, as the project assumed, within the low-noise pump range. The comparative tests have shown that the new low-noise 2PW-SES pumps in almost the whole pressure range emit noise whose level is by as much as $6 \mathrm{~dB}(\mathrm{~A})$ lower than that of the pump of similar design (low-pulsation pump 2). It should be noted that in the low and medium pressure range, the $2 \mathrm{PW}$-SES pump generates noise close to that of the pumps with the profile characterized by zero trapped volume. In addition, this pump can work under higher working pressures than the commercially available low-noise pumps.

\section{Acknowledgements}

This research was carried out in collaboration with the firm PHS HYDROTOR S.A. as part of the project "Development of innovative gear pumps with reduced acoustic emission level" within the framework of the Innovative Economy Operational Programme 2007-2013, Priority 1. Research on and development of modern technologies, Action 1.4 Support of target projects. Project no. POIG.01.04.00-04-345/13.

\section{References}

Bury P. and Osiński P., 2015. Evaluation of coefficient of delivery nonuniformity in newly designed gear pumps with reduced acoustic emission level, (Report series SPR no. 043/2015). Wroclaw University of Science and Technology, Wrocław (in Polish).

Kollek W., 1996. Gear pumps - design and operation. Ossolineum, Wrocław (in Polish).

Osiecki, A., 2004. Hydrostatic drive of machines. Warszawa (in Polish).

Osinski, P., 2014. Modelling and design of gear pumps with modified tooth profile. LAP LAMBERT Academic Publishing, Saarbrücken.

Osiński, P., 2013. High-pressure and low-pulsation external gear pumps. Oficyna Wydawn. Politechniki, Wrocław (in Polish).

Stryczek, S., 2014. Hydrostatic drive. Vol. 1. Wydawnictwo WNT, Warszawa (in Polish).

Szablowski K., 1997. Displacement pumps. PWN, Warszawa-Kraków (in Polish). 